\title{
The outcome of young colorectal cancer patients is controversial
}

\author{
Jian-Fei Fu
}

Received: 30 June 2013/Accepted: 12 July 2013/Published online: 25 July 2013

(C) Springer-Verlag Italia 2013

Yeo et al. [1] report that there was a significant difference in the type of relapse among three age groups. Table 2 in their article shows that $23(31.5 \%), 65$ $(25.3 \%)$ and $409(19.5 \%)$ patients in three age groups had distant metastasis (M1).The authors do not explain the modality of surgery for patients with M1. Moreover, only when a R0 resection was achieved, could the type of relapse be analyzed. Otherwise, the results should be described as disease progress. But Table 2 shows that there were $4(16.7 \%), 14(19.3 \%)$ and $113(28.2 \%)$ patients in three groups with diffuse metastases (multiple locations), and it is not easy to achieve an R0 resection for such patients.

When the multivariate Cox regression analysis was done, the factor of the American Joint Committee on Cancer (AJCC) stage should have been considered as a continuous variant rather than category variant. It was incorrect to put perineural invasion (263 not reported) and vascular emboli (215 not reported), into the Cox model, when so many values were missing.

Finally, I would like to point out a proofing error. There were $65(25.3 \%)$ patients in group 2 and 409 (19.5\%) patients in group 3 with distant metastasis (M1). Why were only $62(24.1 \%)$ patients in group 2 and 407 (19.4\%) patients in group 3 diagnosed with stage IV? Which stage was diagnosed for the other 3 cases in group 2 and 2 cases in group 3 with M1?

Conflict of interest None.

\section{Reference}

1. Yeo SA, Chew MH, Koh PK, Tang CL (2013) Young colorectal carcinoma patients do not have a poorer prognosis: a comparative review of 2,426 cases. Tech Coloproctol. doi:10.1007/s10151-0130977-z
J.-F. Fu ( $₫)$

Oncology Department, JinHua Central Hospital, JinHua City,

Zhejian, China

e-mail: nuifu@sina.com 